\title{
Estratégias pedagógicas na educação a distância: Um olhar a partir de diferentes contextos
}

\section{RESUMO}

O artigo tem como objetivo apresentar o mapeamento, construção e aplicação de estratégias pedagógicas (EP) voltadas para a Educação a Distância (EaD) em diferentes contextos. Para isso, foram utilizados cursos de extensão em áreas distintas, que versaram sobre aspetos socioafetivos da EaD, m-learning e inclusão digital de idosos. A realização da investigação mostrou-se necessária, tendo em vista que o entendimento sobre o conceito de estratégias pedagógicas costuma ser amplo e, muitas vezes, impreciso. Este artigo apresenta uma definição de estratégias pedagógicas, bem como situações de implementação na EaD. As EP podem ser consideradas como um conjunto de ações colocadas em prática pelo professor para alcançar uma finalidade voltada à educação. A metodologia empregada para a realização desta pesquisa foi a qualitativa do tipo estudo de casos múltiplos. A coleta de dados teve como base o desenvolvimento, aplicação e avaliação de estratégias pedagógicas em cursos de extensão. A partir da análise dos dados, foi possível mapear diferentes estratégias e verificar a pertinência da sua adequação, de acordo com o contexto e perfil de cada público. As EP necessitam ser constantemente avaliadas, principalmente em função das transformações que podem ocorrer com a inclusão de novas tecnologias digitais no contexto virtual, além do perfil heterogéneo dos alunos envolvidos no processo educativo.

Palavras-chave: Estratégias pedagógicas;

Educação a Distância

\section{INTRODUÇÃO}

O objetivo do artigo é apresentar o mapeamento, construção e aplicação de estratégias pedagógicas voltadas para a Educação a Distância (EaD) em diferentes contextos. 0 entendimento sobre estratégias pedagógicas (EP) é utilizado de forma abrangente e impreciso. Embora muitos autores adotem o termo, poucos são aqueles que discorrem sobre a definição do conceito e seu real significado. Neste sentido, tendo em vista suas possibilidades e desafios, entende-se que é inviável transpor estratégias pedagógicas das
Leticia Rocha Machado ${ }^{i}$

Ana Carolina Ribeiro Ribeiro $^{\text {ii * }}$

Anna Helena Silveira Sonego ${ }^{\text {iii * }}$

Carla Adriana Barvinski ${ }^{i v}$ **

Cristina Alba Wildt

Torrezzan ${ }^{v}$ *

Deyse Cristina Frizzo

Sampaiovi *

Gislaine Rossetti Madureira Ferreiravii * $^{\text {vi }}$

Patricia Alejandra Behar viii *

Tássia Priscila Fagundes Grande $^{\mathrm{ix} *}$

*Universidade Federal do Rio Grande do Sul, Brasil

**Universidade Federal da Grande Dourados, Brasil 
práticas tradicionais para a Educação a Distância, sobretudo, no que diz respeito às suas possibilidades e desafios. Portanto, conhecer os aspetos tecnológicos e sua aplicabilidade poderá permitir ao professor a possibilidade de proporcionar aos alunos espaços eficazes de construção do conhecimento. No entanto, para um melhor emprego dessas possibilidades, é importante investigar alternativas para desenvolver e aplicar EP com diferentes públicos no contexto da EaD. Assim, com base no panorama delineado a seguir, são apresentadas algumas potencialidades para desenvolver e aplicar EP em diferentes cenários e públicos. Na terceira seção, é problematizado o conceito de estratégias pedagógicas, especificamente, na EaD. Na quarta seção, é apontada a metodologia do presente estudo e, na quinta, a análise de dados coletados em diferentes contextos, tendo, como foco, o mapeamento e a avaliação de estratégias pedagógicas. Por fim, na seção seis, são evidenciadas as considerações finais.

\section{EDUCAÇÃO A DISTÂNCIA: POSSIBILIDADES EDUCACIONAIS}

O panorama atual aponta para um novo perfil de sociedade, no qual a população está cada vez mais acedendo a, e adquirindo, aparelhos eletrónicos para atividades de lazer, trabalho e, inclusive, fins educacionais. Entre as tecnologias mais consumidas e utilizadas, estão os dispositivos móveis (DM) como, por exemplo, os smartphones e tablets. Uma pesquisa realizada pela Universidade Fundação Getúlio Vargas (Meirelles, 2021), relatou que, atualmente, existem 230 milhões de smartphones sendo utilizados no Brasil e, adicionando as quantidades de notebooks e tablets, verifica-se um total de 324 milhões de dispositivos portáteis estão em uso. Esse panorama possibilita uma maior discussão no âmbito educacional sobre o uso desses tipos de recursos em sala de aula, bem como benefícios e limitações presentes na sua utilização. Conforme Sales et al. (2017), as pessoas, hoje em dia, vivem numa sociedade dinâmica, na qual realizam muitas tarefas simultaneamente, além de usar várias tecnologias digitais para se comunicar. No entanto, além dos jovens e adultos, os idosos estão incluindo-se digitalmente, buscando acompanhar essas mudanças através de cursos formais e informais que priorizam uma educação permanente (Viana \& Peralta, 2020). Neste sentido, entende-se que uma alternativa para atender a essa demanda é através da Educação a Distância (EaD) e da m-learning.

A EaD é uma modalidade já estabelecida e fortalecida no âmbito nacional e internacional que se caracteriza pela mediação das tecnologias digitais que apoiam as relações e o processo de ensino e aprendizagem em diversas áreas do conhecimento (Cavalcante Filho et al., 2020). Essa modalidade tem-se expandido cada vez mais em decorrência de vários fatores, como a possibilidade de o aluno realizar uma graduação ou cursos informais de educação permanente sem precisar frequentar diariamente e presencialmente uma instituição de ensino. Para tanto, ele pode realizar aulas em qualquer lugar e a qualquer momento através/com o uso de tecnologia digital (Cavalcante Filho et al., 2020).

Já a m-learning pode ser considerada uma modalidade de educação que perpassa a EaD, pois é definida como o uso de tecnologias móveis combinadas, ou não, no intuito de possibilitar a mobilidade da aprendizagem (Matoski et al., 2020). Dessa forma, entende-se que existe um amplo potencial para a aplicação dos dispositivos móveis no processo de ensino e aprendizagem virtual. Assim, pode-se observar que, na Educação a Distância, 
são empregadas diferentes tecnologias digitais (móveis ou não), bem como recursos como os disponibilizados nos ambientes virtuais de aprendizagem. No entanto, é necessário o planeamento de metodologias e estratégias pedagógicas condizentes, já que cada aluno apresenta um perfil, o que determina a necessidade de novas investigações. Sob essa ótica, os aspetos socioafetivos influenciam diretamente o processo de ensino e aprendizagem, pois estão atrelados às interações sociais realizadas nos AVA. Marques et al. (2019, p. 37) ainda apontam que os aspetos socioafetivos "[...] podem ser aprendidos ou aprimorados intencionalmente, aumentando os recursos internos [...]". Em outros termos significa desenvolver estratégias para a EaD que permitam essas construções, que são primordiais para uma educação emancipatória que viabilize a aprendizagem para o convívio em sociedade. Dessa forma, os aspetos mencionados apresentam-se como características que devem ser consideradas ao planear e desenvolver EP para cursos a distância, já que são essenciais para a construção do conhecimento. Logo, é necessário repensar os modelos de ensino e aprendizagem que estão sendo adotados na atualidade no intuito de apresentar e desenvolver ações que favoreçam um maior engajamento dos alunos na EaD.

\section{ESTRATÉGIAS PEDAGÓGICAS PARA A EDUCAÇÃO A DISTÂNCIA}

O termo "estratégia pedagógica" (EP) está vinculado com muitos outros, entre eles, é possível citar: estratégias de aprendizagem, estratégias educacionais, estratégias de ensino e aprendizagem. No entanto, ao serem analisadas publicações sobre EP, pode-se verificar que alguns elementos são fundamentais para elas, como, por exemplo, o paradigma educacional norteador, seu contexto (totalmente virtual ou híbrido), os recursos tecnológicos que podem ser utilizados no AVA e/ou fora dele para sua aplicação e os processos educacionais necessários (métodos, ações etc.) (Masetto, 2003; Amaral, 2017; \& Behar et al., 2019). Assim, as EP devem ser flexíveis, ou seja, modificarem-se no decorrer do processo de ensino e aprendizagem. Masetto (2003) aponta a necessidade de o professor pensar em estratégias que facilitem o processo de ensino e aprendizagem em sala de aula, empregando, para tanto, materiais diversificados como os digitais, técnicas de ensino, ações educacionais etc. 0 mesmo autor ainda salienta que, nas EP, é necessário: (a) utilizar as mais adequadas para cada objetivo pretendido da prática pedagógica; (b) dispor de estratégias apropriadas para cada sujeito ou grupo de alunos; (c) adotar uma variedade de estratégias que sejam flexíveis no decorrer do processo. Portanto, as EP podem ser definidas como "[...] ações e as formas para se alcançar uma finalidade voltada à educação" (Amaral, 2017, pp. 12-13). Behar et al. (2019, p. 16) aproximam-se desse conceito e ampliam-no, ponderando que as estratégias "[...] podem ser compreendidas como um conjunto de ações educacionais. [...] As estratégias pedagógicas podem ser sugestões para usar novas tecnologias digitais; aplicações de atividades complementares [...]" (Behar et al., 2019, p. 16). Dessa forma, o conceito utilizado neste estudo será o de Amaral (2017) e Behar et al. (2019), que consideram as EP como um conjunto de ações, técnicas, métodos, recursos articulados para fins educacionais.

As estratégias podem ser aplicadas em muitos contextos, práticas e públicos, como é o caso de adultos e idosos. Na Educação a Distância, Souza et al. (2008) salientam que, como a mediação do processo educativo a distância geralmente ocorre através de um Ambiente Virtual de 
Aprendizagem, é pertinente dispor de ferramentas que possibilitam a comunicação síncrona e assíncrona entre os sujeitos. Desse modo, a implementação de estratégias pedagógicas que promovam o diálogo e a participação dos alunos deve ser viabilizada na EaD. Assim sendo, além de promover a construção de competências relacionadas à comunicação por todos os atores, também auxilia na criação de espaços de interação e de construção coletiva, através de possibilidades concretas de trabalho colaborativo. Portanto, pensar nesses aspetos torna-se importante, pois cada ferramenta exige uma série de habilidades distintas "e propiciam diferentes estratégias pedagógicas, que exigem participação em tempo real ou diferida, possibilitando a expressão, a intervenção e a colaboração para a construção coletiva do conhecimento" (Souza et al., 2008, p. 331). O professor convive com pessoas e com grupos diferentes, o que se torna um desafio diário, sendo necessário refletir e promover estratégias que busquem atentar e compreender valores e características tão diferenciadas entre os alunos. No intuito de analisar estes diferentes cenários, a seguir é apresentada a metodologia de pesquisa.

\section{METODOLOGIA}

Para a realização desta pesquisa, adotou-se uma abordagem qualitativa do tipo estudo de casos múltiplos. 0 público-alvo foi composto por professores em formação continuada ou permanente que participaram, ao longo do ano de 2018, de cursos de extensão oferecidos numa instituição pública no sul do Brasil. O processo de elaboração deste estudo envolveu três etapas. Na primeira etapa, foram criadas, com base na experiência prévia docente e em referencial teórico, estratégias pedagógicas preliminares distintas para cada estudo de caso. Esse processo ocorreu em discussões semanais pelos autores que participam do grupo de pesquisa (Núcleo de Tecnologia Digital Aplicada à Educação - NUTED da Universidade Federal do Rio Grande do Sul/Brasil) e possuem formação e experiência docente na $\mathrm{EaD}$, em contextos distintos, há mais de cinco anos. Em seguida, na etapa 2 (dois), as EP foram aplicadas em três estudos de caso, cada um deles abordando diferentes conteúdos: aspetos socioafetivos, aprendizagem móvel e construção de materiais educacionais digitais por idosos. Na sequência, na etapa 3 (três), foram realizadas a interpretação dos dados coletados, o aprimoramento e a validação das estratégias pedagógicas elaboradas. Cabe salientar que, no decorrer da criação das estratégias pedagógicas, também foi necessário, além de sua avaliação constante, o desenvolvimento de novas EP que pudessem subsidiar e tornar flexível a prática pedagógica adotada em cada etapa. Desse modo, a seguir, são detalhadas essas etapas.

\subsection{ETAPA 1: ELABORAÇÃO DE ESTRATÉGIAS PEDAGÓGICAS PARA O ENSINO HÍBRIDO}

Como já abordado, existe uma diversidade de definições relacionadas às estratégias pedagógicas. Assim, foi necessário realizar um levantamento teórico de conceitos, principalmente os voltados para a EaD. Para a constituição da revisão sistemática, foram consultados sites de periódicos como do CAPES ScienceDirect e livros publicados sobre a temática, os quais, assim como a experiência docente dos pesquisados, auxiliaram na construção das estratégias pedagógicas preliminares, já que se apoiaram no delineamento dos elementos necessários para a sua elaboração. Dessa forma, 
foram desenvolvidas estratégias pedagógicas para cada estudo de caso no formato textual, visando abranger alguns elementos para o seu desenvolvimento como: Paradigma educacional: para conduzir a construção de cada EP, foi necessário delinear o paradigma educacional que iria guiar 0 desenvolvimento das práticas pedagógicas, sendo adotado, neste estudo, o construtivista; Contexto: para que as EP fossem empregadas na prática pedagógica, foi pertinente apontar o público-alvo (necessidades específicas), assim como contemplar as necessidades inerentes da modalidade EaD (virtual ou híbrida), sendo que, ao todo, foram delimitados três contextos distintos (estudos de caso); Processos educacionais: foram definidos como sendo as ações práticas que o docente poderia aplicar no contexto e perfil delineado, considerando os outros elementos da EP (recursos tecnológicos e métodos); para cada EP, foram apontadas uma ou mais tecnologias dentro do AVA e/ou fora dele que poderiam ser aplicadas ou utilizadas no processo, sendo necessário considerar os procedimentos educacionais empregados para as necessidades do cenário definido. Destaca-se que outros elementos podem surgir e devem ser considerados para o desenvolvimento de uma estratégia pedagógica. No entanto, para a construção das EP deste estudo, apenas os elementos anteriormente citados foram contemplados, sendo desenvolvidas estratégias preliminares que foram utilizadas, avaliadas e validadas nas etapas conseguintes. Cabe destacar que, além das estratégias iniciais, foi necessário, também, construir novas estratégias visando ao suporte ao processo de ensino e aprendizagem em cada um dos contextos da EaD.

\subsection{ETAPA 2: APLICAÇÃO DAS ESTRATÉGIAS PEDAGÓGICAS EM CURSOS DE EXTENSÃO}

Com o objetivo de avaliar as estratégias criadas, foram ofertados, na Universidade, três cursos de extensão especificamente para esse propósito. Neste sentido, foram desenvolvidas EP com foco na modalidade EaD, que contemplassem as necessidades no que diz respeito aos aspetos afetivos e sociais, o uso da m-learning e, por fim, o desenvolvimento de materiais educacionais digitais por idosos. Essa abordagem foi utilizada para contemplar contextos e perfis de alunos distintos em cada curso, objetivando avaliar a utilização das estratégias em diferentes cenários e também as necessidades dos participantes dessa modalidade de ensino. Porém, tendo em vista a necessidade do rigor científico para a condução dos estudos de casos, adotaram-se padrões para a estruturação das estratégias pedagógicas e para os procedimentos de coleta, análise e interpretação de dados. Assim, os estudos de caso foram três:

\section{Caso 1 - Curso 1}

Foi realizado, no segundo semestre de 2018, tendo como objetivo abordar os aspetos socioafetivos para EaD. 0 curso foi organizado em dez aulas entre presenciais e a distância, contando com carga-horária de 40 horas. 0 públicoalvo consistiu em 22 professores, todos com experiência em EaD, sendo 91\% do género feminino e $9 \%$ do masculino. Para a sua realização foi utilizado o AVA ROODA (Rede c00perativa De Aprendizagem) que tem como objetivo proporcionar um espaço de interação entre seus participantes, em especial, as ferramentas Mapa Social e Mapa Afetivo. Participaram professores que possuíam algum interesse em trabalhar e discutir sobre o tema. Assim, foram propostas estratégias visando fomentar os dois aspetos: o social e o afetivo. 
Caso 2 - Curso 2

Teve como objetivo discutir e refletir sobre o uso dos dispositivos móveis a fim de propor estratégias para a m-learning. Foi ofertado para professores da educação básica pertencentes à rede pública estadual e municipal de Porto Alegre e região metropolitana. Participaram 14 docentes, sendo 13 do género feminino e um do masculino, todos com interesse em implementar atividades com os dispositivos móveis.

\section{Caso 3 - Curso 3}

Consistiu na realização de um curso com o objetivo de inclusão digital de idosos, voltado para a construção de materiais digitais como vídeos, sites etc. Participaram 14 pessoas com idade entre 60 e 80 anos. Nesse contexto, foram realizadas atividades que abordaram essa temática através de ferramentas de interação e comunicação.

Para a coleta de dados, foram utilizados diferentes instrumentos que possibilitaram uma triangulação dos resultados no intuito de propiciar a avaliação e reestruturação das estratégias previamente elaboradas e ainda a criação de novas. Após o término dos cursos, foram disponibilizados questionários avaliativos sobre as estratégias adotadas, bem como a possibilidade de sugestões de outras possíveis ações. Os questionários possuíam perguntas distintas, sendo semelhantes no quesito que indagava sobre a avaliação das EP aplicadas.

O AVA utilizado em todos os cursos foi o ROODA e suas ferramentas de interação e comunicação: Biblioteca - espaço utilizado para a publicação e a organização de materiais e links que apresenta aos alunos a possibilidade de inserção de materiais e de comentários nos materiais inseridos; Contatos - que consiste numa lista com nome e e-mail dos alunos, professores e tutores e permite o envio de mensagens que são enviadas para o e-mail pessoal de cada usuário; Bate-papo - espaço de comunicação síncrona entre os usuários, sendo as conversas visíveis para todos da turma que estejam conectados; Fórum - espaço de interação assíncrona entre os usuários, através da criação de tópicos e de mensagens; Webfólio - sistema de envio de arquivos que propicia a sua publicação e a organização, permitindo a inserção de comentários por professores, tutores e colegas nos materiais postados.

\subsection{ETAPA 3: AVALIAÇÃO E VALIDAÇÃO DAS ESTRATÉGIAS PEDAGÓGICAS PARA EAD}

Nessa etapa, foi possível realizar a análise final sobre todas as etapas anteriores. Para a coleta de dados, no decorrer dos estudos de casos nos cursos oferecidos, foram utilizados diferentes instrumentos que possibilitaram uma triangulação dos resultados no intuito de apresentar estratégias pedagógicas para a EaD: Questonário, observação participante e produções, postagens e ferramentas do ambiente virtual de aprendizagem.

Questionário: foram aplicados questionários ao término de cada etapa da pesquisa, em que foi possível analisar e avaliar as estratégias pedagógicas propostas e adotadas; 
Observação participante: no decorrer do processo, os pesquisadores atuaram como mediadores nos cursos e disciplinas, o que possibilitou que anotações fossem realizadas e adicionadas na discussão dos dados coletados;

Produções, postagens e ferramentas do ambiente virtual de aprendizagem adotado: foram coletadas as informações registradas pelos participantes no Ambiente Virtual de Aprendizagem (AVA), principalmente, aquelas relacionadas com as EP adotadas nos cursos.

$\mathrm{Na}$ análise dos dados coletados nos instrumentos de pesquisa, foram utilizadas as etapas sugeridas por Bardin (2010) referentes à análise de conteúdo. Os dados analisados viabilizaram a proposição de estratégias específicas para cada uma das categorias, desenvolvidas nos três estudos de caso descritos. As categorias elencadas foram: Aspeto Social e EaD, Aspeto Afetivo e EaD, m-learning e inclusão digital de idosos. Assim, com base nessas aplicações, foi possível planear e propor estratégias para $\mathrm{EaD}$, conforme os resultados apresentados a seguir.

\section{RESULTADOS}

O objetivo desta investigação foi mapear, construir e aplicar estratégias pedagógicas voltadas para a Educação a Distância em diferentes contextos, através da realização de estudos de caso. Neste sentido, para a finalidade de análise, essas estratégias foram categorizadas em: Aspetos Sociais e EaD; Aspetos Afetivos e EaD; m-learning e público idoso, conforme será apresentado a seguir. Dessa forma, os dados coletados serão apresentados separadamente, visando a uma melhor análise deles.

\subsection{ESTRATÉGIAS PEDAGÓGICAS: UM FOCO NOS ASPETOS SOCIAIS NA EAD}

O curso realizado na Etapa 2 (dois) possibilitou abordar os aspetos sociais e afetivos de forma conjunta e em aulas separadas. Assim, visando a uma melhor análise dos dados são tratadas, primeiramente, as estratégias pedagógicas com foco nos aspetos sociais e, posteriormente, nos aspetos afetivos. A construção de estratégias com foco nos aspetos sociais ocorreu com base na ferramenta Mapa Social. O Mapa Social é uma funcionalidade que propicia, a partir das interações dos usuários nas ferramentas de comunicação do ROODA, a geração de sociogramas que permitem, de forma visual, acompanhar a relações estabelecidas no AVA. Portanto, através do sociograma, é possível identificar vínculos, influências e preferências que existem dentro de um grupo. A partir das interações sociais observadas, ao longo do tempo em disciplinas de graduação e cursos de extensão, identificaram-se diferentes perfis dos sujeitos no ambiente virtual de aprendizagem, sendo possível distinguir Indicadores de Interação Social. Assim, com base nessas análises, foi possível apontar determinadas características recorrentes nas trocas sociais realizadas no ambiente, ou seja, as formas em que os diferentes perfis do sujeito social podem prevalecer (Behar et al., 2019):

- Colaboração - indica o quanto o sujeito contribui com as interações da turma e também através de postagem de materiais. 
- Grupos informais - apresenta as trocas constantes estabelecidas entre três ou mais sujeitos através das funcionalidades do ROODA, constituindo um agrupamento.

- $\quad$ Popularidade - indica quais sujeitos destacam-se por manter uma frequência maior de interações do que o restante dos participantes da turma.

- Distanciamento pela turma - indica o sujeito que entra em contato com os colegas e não recebe retorno. Dessa forma, possibilita ao professor visualizar casos em que um aluno é isolado pelo restante da turma.

- $\quad$ Ausência - refere-se ao sujeito que entra no ambiente e não retorna às solicitações de contato da turma.

Conforme Souza (2012), um ambiente que seja propício à aprendizagem colaborativa deve prover meios de interação e de comunicação. Nesse contexto, entende-se que um ambiente que disponha de ferramentas de interação, aliado a procedimentos metodológicos e organizacionais que possam prever a utilização desses espaços de forma ampla e diversificada tende a contribuir de maneira significativa para o processo educativo. 0 acompanhamento dessas interações também é importante tendo em vista que, nesse processo, evidenciam-se comportamentos e práticas que podem auxiliar o professor a compreender as dinâmicas que se estabelecem entre os alunos. Assim, é possível a visualização do direcionamento das trocas, bem como casos de distanciamento social e ausência de relações que são, muitas vezes, determinantes para a permanência ou evasão dos alunos nos cursos virtuais. Portanto, ao observar as relações que se estabelecem numa turma, entende-se que o professor tem a oportunidade de promover estratégias que visem diminuir as distâncias e aprimorar as relações de colaboração e reciprocidade. Neste sentido, participaram deste estudo de caso, realizado num curso de extensão, 14 alunos com experiência em EaD que foram convidados a avaliar e sugerir possíveis novas estratégias pedagógicas voltadas para a promoção das interações sociais para cada um dos indicadores citados anteriormente. Dessa forma, foi desenvolvido um banco com 70 estratégias. A seguir, são apresentados alguns exemplos de EP a partir dos Indicadores:

- Indicador Colaboração através da ferramenta Fórum: "Motivar o debate sobre temas relevantes em uma ferramenta como o fórum pode fazer com que a turma aumente sua participação e, consequentemente, sua colaboração entre membros. Trata-se de uma forma de discussão, na qual os alunos podem participar sem necessidade de disponibilizarem-se conectados ao mesmo tempo ou no mesmo lugar" (E1).

- Indicador Popularidade através da ferramenta Webfólio: “É uma possibilidade pedir que os alunos que se destacam no quesito de popularidade utilizem o Webfólio para postar os arquivos e entrem em contato com os demais alunos, comentando em suas atividades também, estimulando-os a enviar respostas sobre suas atividades aos colegas" (E2).

- Indicador Grupos informais através da ferramenta Webfólio: "Os grupos informais podem traduzir muito sobre a realidade dos/as alunos/as. Quais afinidades formam o grupo desses alu- 
nos/as? Estimular trabalhos em grupos e/ou grupos de estudos formalizando esses grupos pode ser produtivo. 0 Webfólio pode ser útil nesse sentido" (E3).

- Indicador Ausência na ferramenta Bate-papo: "Tentar entrar em contato com o aluno por uma ferramenta cuja resposta é mais instantânea como o bate-papo pode contribuir para chamar sua atenção de volta ao ambiente virtual e promover trocas mais velozes, para gerar interesse tanto no ambiente virtual, como nos conteúdos estudados. A demora em responder pode gerar 0 desinteresse no aluno e fazê-lo afastar-se do AVA até caracterizar sua ausência de interação e seu isolamento e desinteresse sobre os demais membros da turma" (E4).

- Indicador Distanciamento através do Diário de Bordo: "O diário de bordo permite sondar os sentimentos do aluno, a partir das suas postagens é possível saber como o aluno se sente com relação ao isolamento social e, assim, o docente poderá intervir com o restante da turma" (E5).

As estratégias foram avaliadas, padronizadas e expandidas, a partir desse primeiro levantamento, de forma a construir um banco ainda maior de EP com foco em interações sociais. Nas estratégias apresentadas, pode-se observar que os recursos de comunicação tiveram destaque como condutor em cada EP, uma vez que, através deles, será possível estabelecer relações sociais no AVA. Também é pertinente considerar que as estratégias foram apontadas de forma ampla no intuito de contemplar as necessidades mais recorrentes na modalidade $\mathrm{EaD}$, conforme a experiência dos participantes do curso. Dessa forma, o estudo subsidiou a criação de 144 estratégias pedagógicas com base em aspetos sociais que foram avaliadas por especialistas, aprimoradas e disponibilizadas no ambiente virtual utilizado (Ribeiro, 2019). 0 intuito foi abordar os diferentes públicos da EaD e possibilitar ao professor formas de repensar a sua prática realizada à distância. Essas estratégias também podem servir como base para analisar se os ambientes virtuais de aprendizagem estão promovendo espaços que auxiliem no estabelecimento e fortalecimento das interações sociais.

\subsection{ESTRATÉGIAS PEDAGÓGICAS: UM FOCO NOS ASPETOS AFETIVOS DA EAD}

Para o mapeamento e avaliação das estratégias pedagógicas voltadas para os aspetos afetivos foi utilizado o mesmo curso apresentado anteriormente. $\mathrm{Na}$ parte do curso destinada ao estudo dos aspetos afetivos, os 14 participantes tiveram dois momentos: Primeiro, avaliaram estratégias já desenvolvidas por Longaray (2014) e, no segundo, propuseram novas possibilidades de aplicação na EaD. Para complementar a análise dos dados, foi empregado o Mapa Afetivo que é uma funcionalidade do ambiente virtual de aprendizagem ROODA, plataforma usada para aulas de Educação a Distância, na instituição em que esta pesquisa foi desenvolvida. O Mapa Afetivo possibilita ao professor verificar os estados de ânimo e os traços de personalidade dos estudantes que são identificados a partir dos textos e interações produzidos pelos alunos no AVA. Assim, as estratégias estão atreladas à subjetividade afetiva expressa pelo estudante em seus textos produzidos nas ferramentas de comunicação do ROODA e que são sugeridas ao professor, de acordo com a demanda emocional do aprendiz. Nesse cenário, ao todo, foram avaliadas 20 
estratégias pedagógicas relacionado ao afetivo: “A autoavaliação do trabalho de formador é importante para diagnosticar propostas instigantes para os alunos" (E1); "A satisfação pode aumentar se o aluno for parabenizado pelas contribuições à turma (quadrante insatisfeito e satisfeito)" (E2); "Os materiais de apoio como artigos científicos, notícias, objetos de aprendizagem, capítulos de livro, áudios, vídeos, podem auxiliar o aluno a aumentar seu interesse sobre os temas" (E3); "Os feedbacks das tarefas podem provocar um maior envolvimento do aluno nas aulas virtuais" (E4); "As ferramentas de comunicação, tais como Bate-papo, Fórum, Diário de Bordo e Contatos, são ótimos recursos para resgatar o aluno ausente" (E5); "A utilização de materiais complementares pode proporcionar novas vivências e descobertas, enriquecendo o processo de aprendizagem do aluno com o auxílio de materiais multimídias" (E6); "O uso de ferramentas ou recursos multimídia diversos (tais como, vídeos, músicas, textos, hipertextos, websites etc.) pode abranger os diferentes estilos de aprendizagem dos alunos" (E7); "As ações propostas podem incentivar as interações interpessoais como uma estratégia que promove o envolvimento daqueles alunos que pouco atuam na aula" (E8); "O feedback do formador pode auxiliar o aluno a melhorar sua atuação frente aos desafios propostos" (E9); "Os laços afetivos com o aluno podem ser estreitados através da interação frequente no AVA, procurando mantê-lo interessado nas aulas" (E10); "A autoavaliação pode propiciar uma reflexão do aluno sobre sua atuação nos AVA e também indicar possíveis ferramentas e recursos digitais que mais atraem os alunos" (E11); "A indicação de cursos de extensão, palestras, oficinas e eventos relacionados aos temas tratados em aula pode despertar o interesse do aluno" (E12); "O diário de bordo é uma ótima ferramenta para compreender as dificuldades dos alunos" (E13); "Os materiais de apoio, como artigos científicos, notícias, objetos de aprendizagem, capítulos de livro, áudios, vídeos etc., podem auxiliar o aluno a aumentar seu interesse sobre os temas abordados em sala de aula" (E14); "Os conhecimentos prévios dos alunos devem ser considerados e o professor pode (re)planejar e conduzir melhor a sua aula" (E15); "O feedback para o aluno deve ser o mais rápido possível para haver maiores chances de ele continuar realizando as atividades" (E16); "O conteúdo deve ser apresentado em medias diferentes para instigar a curiosidade do aluno" (E17); "O diário de bordo é uma ferramenta importante para verificar se é necessário intervir, ou não, na manifestação dos estados afetivos" (E18); "O processo de aprendizagem deve ser acompanhado e mediado, no intuito de fornecer feedback ao aluno para incentivá-lo a uma participação mais ativa" (E19); "O uso de ferramentas ou recursos multimídia diversos (tais como vídeos, músicas, textos, hipertextos, websites etc.) pode abranger os diferentes estilos de aprendizagem dos alunos" (E20).

As EP foram avaliadas como "bom", "muito bom" ou "excelente" pelos sujeitos da pesquisa. Dessa forma, foi possível observar que todas EP foram bem avaliadas, no entanto algumas destacaram-se como a EP3, que foi a mais bem avaliada no Estado de ânimo Satisfeito e Animado (95\%). A estratégia EP1 foi avaliada como "bom" por 9\% dos participantes. Já no estado de ânimo Insatisfeito, a EP8 e a EP11 foram as mais bem avaliadas, sendo 77\% de respostas em ambas estratégias. Já a EP7 e EP13 foram avaliadas como "regular" por 4,5\% dos sujeitos em ambas as estratégias. Por último, no que tange às estratégias do estado de ânimo Desanimado, a EP19 e a EP20 foram as mais bem avaliadas com $81 \%$ dos sujeitos apontando como 
"excelente". A EP16 foi avaliada como "regular" por 4,5\% dos sujeitos. Assim, pode-se observar que as estratégias que remetem à avaliação, ao incentivo e à disponibilidade de materiais de apoio compõem as mais bem avaliadas em todos os estados de ânimos. Já as que foram avaliadas como "regular" são aquelas estratégias que apresentam características gerais, sem haver exemplos de aplicação. Portanto, é necessário que novas reformulações sejam realizadas no intuito de contemplar outras especificidades e contextos diversificados.

\subsection{ESTRATÉGIAS PEDAGÓGICAS: UM FOCO NA M-LEARNING}

A avaliação das estratégias para a m-learning ocorreu durante a realização de um curso de extensão sobre a aprendizagem móvel. No curso, os 14 professores tiveram três momentos envolvendo as estratégias pedagógicas com foco em m-learning. No primeiro, os professores tomaram conhecimento e discutiram o conceito de estratégias, sua relevância, a criação e implementação na sala de aula. No segundo momento, foram apresentadas algumas estratégias pedagógicas utilizadas com potencial para serem implementadas no âmbito da aprendizagem móvel: trabalho colaborativo e resolução de testes (atividades: ferramenta de apoio e a discussão de conteúdos de modo presencial e a distância através dos dispositivos móveis). Para o terceiro momento, os alunos do curso, em duplas, analisaram as referidas estratégias e realizaram a avaliação através de um questionário. Neste aspeto, foram indagados sobre as estratégias pedagógicas adotadas pelos participantes para desenvolver a m-learning, bem como sobre a sua avaliação. Como resposta, obteve-se uma autoavaliação e reflexão sobre as estratégias apresentadas por parte dos professores que realizaram o curso. Portanto, através dessa prática, verificaram-se as seguintes estratégias pedagógicas voltadas à aprendizagem móvel: "Jogos Educacionais: Usar jogos educacionais em forma de aplicativos nos dispositivos móveis" (E1); "Ferramentas de comunicação: Utilizar ferramentas de comunicação como WhatsApp para estreitar os laços sociais e afetivos" (E2); "Situaçõesproblemas: Apresentar situações-problemas contextualizadas com as características da mobilidade" (E3); "Pesquisa na internet: Propor desafios que possibilitem a pesquisa através dos dispositivos móveis" (E4); "Aplicativos: Utilizar aplicativos diversificados tanto off-line como online" (E5); "Materiais de apoio: Disponibilizar materiais de apoio responsivos para DM" (E6).

Assim, as EP anteriormente listadas foram avaliadas no quesito adequação. Neste sentido, os participantes expressaram que: "Sim! Há necessidade do professor colocar-se disponível em aprender a usar e planejar seguindo os preceitos apontados" (P1); "Sim, pois a aula se torna mais atrativa para o aluno" (P2); "Sim, pois contempla e abrange possibilidades de uso dos Dispositivos Móveis" (P3); "Sim, pois pode ser usada como base para o planejamento em determinada área, sendo possível adaptações de acordo com os conteúdos que serão trabalhados" (P5). Com base nas falas, pode ser visualizado que delinear estratégias pedagógicas com foco na m-learning pode contribuir na ampliação do alcance dos estudantes durante o processo de construção de conhecimento, assim como de conteúdos que podem ser abordados a partir do uso dos dispositivos móveis. Assim, as EP auxiliam no planeamento e implementação de atividades na educação a distância e também de forma presencial. 


\subsection{ESTRATÉGIAS PEDAGÓGICAS: UM FOCO NO PÚBLICO IDOSO}

No intuito de desenvolver e analisar estratégias pedagógicas para o público idoso na EaD, foram realizadas avaliações num curso de extensão virtual ofertado na Unidade de Inclusão Digital de Idosos (UNIDI), de uma Universidade pública do Brasil. 0 curso objetivou preparar os idosos para atuarem como multiplicadores na EaD. Para isso, os participantes construíram Materiais Educacionais Digitais (MED), seguindo orientação e supervisão de uma professora e duas monitoras. Para tanto, foram organizadas 15 aulas com estratégias pedagógicas, que tinham como objetivo auxiliar os idosos a definir quais temas poderiam ser abordados em seus respetivos MED. 0 desenvolvimento ocorreu de forma presencial e a distância. Para iniciar, foi apresentada a proposta do curso, bem como realizadas algumas atividades para auxiliar os idosos a pensar em temas de seu interesse. Para isso, foram propostas as seguintes estratégias pedagógicas (EP):

Autorretrato: essa EP teve como objetivo a realização de um autorretrato em uma folha de papel, na qual os idosos apontaram, através de desenhos, as características que consideravam relevantes para tornarem-se multiplicadores como, por exemplo, o que é aprender e ensinar(E1);

Tabela de assuntos de interesse: essa EP foi proposta para que os idosos preenchessem uma tabela que apresentava três colunas com assuntos de seu interesse e possíveis materiais que poderiam desenvolver. Assim, foi possível que os alunos-idosos pudessem visualizar graficamente os possíveis conteúdos de suas aulas (E2);

Fórum de dúvidas: o uso dessa ferramenta possibilitou que os participantes pudessem trocar experiências sobre como estavam pensando em criar suas aulas, bem como suas atividades e MED (E3);

Diário de bordo das vitórias e angústias: essa funcionalidade foi aplicada como uma possibilidade de auxiliar os idosos a compartilhar sentimentos e emoções que emergiram durante o processo. Essas trocas permitem que o professor e/ou tutor possa analisar as dificuldades pontuais de cada participante (E4)

Esquema para a criação das aulas: nesta etapa, os alunos foram instigados a modelar, em forma de esquema, a estrutura que seria desenvolvida em suas aulas, contemplando conteúdos, atividades e materiais complementares (E5)

Webfólio e meus arquivos: a ferramenta foi utilizada para que os participantes pudessem postar e comentar sobre a atividade autorretrato, em âmbito pessoal e em relação ao dos colegas. Essas trocas possibilitaram novas perspetivas sobre suas produções e o redesenho das oficinas propostas (E6). 
Além das estratégias elencadas, outras foram utilizadas, como o uso de ferramentas específicas de construção de MED, aulas sobre pesquisa no Google, sites de imagens livres, apresentações sobre os direitos autorais, recursos para construção de sites/páginas da internet (http://weebly.com), editor de vídeo (VivaVídeo) e serviço de armazenamento e sincronização de arquivos (Google Drive). Assim, após a utilização das EP, essas estratégias foram avaliadas pelos idosos. Na EP sobre o "Autorretrato", seis participantes $(n=14)$ marcaram "Medianamente importante", enquanto quatro $(n=14)$ apontaram como "Extremamente importante". Na EP de "Tabela de assuntos de interesse", quatro participantes $(n=14)$ classificaram como "Muito importante" a pontuação para a atividade da tabela de interesses, enquanto seis $(n=14)$ pontuaram como "Extremamente importante" a EP. Já sobre a EP "Esquema para a criação das aulas", nove participantes (n=14) deram pontuação "Extremamente importante" e três $(n=14)$ elencaram como "Medianamente importante". Desse modo, pode-se observar que todas as estratégias pedagógicas foram bem avaliadas pelos idosos, o que denota a eficiência das EP de acordo com suas finalidades. As outras estratégias pedagógicas também foram positivamente avaliadas, sendo sinalizado apenas a dificuldade em utilizar, de forma técnica, as ferramentas e funcionalidades do AVA empregado. Assim, observa-se que as EP utilizadas durante as aulas contribuíram para os idosos na organização de seus Materiais Educacionais Digitais (MED) e planeamento de suas oficinas. Um dos idosos ainda apontou:

As aulas foram bem explicadas é que, para nós, alunas foi e é bem difícil entender bem como funciona uma oficina. Mas devido às aulas que foram bem elaboradas pelas professoras deu pra mim entender um pouquinho, e tentar desenvolver com o meu pouco conhecimento esse trabalho. Mais umas aulas acho que fica mais claro pra nós. (P1)

A análise dos resultados da pesquisa indica que os idosos consideram as estratégias como estruturas organizadas e delimitadas de forma que possibilitaram uma visualização melhor da oficina que estavam construindo. Já as estratégias com maior possibilidade de reflexão, sem delimitações, não auxiliaram tanto no processo de criação dos MED. Portanto, os estudos de caso permitiram construir e mapear as necessidades emergentes de cada estudo de caso (cenário e público distinto). Pode-se observar que, no que diz respeito aos aspetos socioafetivos, foi necessária uma estrutura que possibilitasse as trocas sociais e o uso de recursos comunicacionais no AVA (e fora dele) para que eles fossem abordados ao longo do processo de ensino e aprendizagem na EaD. Já no que se refere às EP para m-learning, assim como as utilizadas com o público idoso, pode-se notar que as estratégias eram mais vinculadas ao uso e à aplicação de tecnologias digitais. No final, ao todo, foram mapeadas e avaliadas 102 estratégias pedagógicas, sendo 70 relacionadas ao aspeto social, 20 ao aspeto afetivo, seis para m-learning e seis para idosos com foco no desenvolvimento de materiais digitais. Dessa maneira, entende-se que este estudo pode contribuir no que diz respeito a uma reflexão sobre as particularidades de cada cenário abordado, assim como as possibilidades relacionadas a uma avaliação contínua pelo docente sobre suas práticas pedagógicas na EaD. 


\section{CONSIDERAÇÕES FINAIS}

O objetivo deste artigo foi apresentar o mapeamento, construção e aplicação de estratégias pedagógicas voltadas para a Educação a Distância (EaD) em diferentes contextos. As estratégias são relevantes para auxiliar nos panejamentos educacionais como uma ação que guie e oriente os professores, do mesmo modo, contribuindo para o desenvolvimento do processo de ensino e aprendizagem em diferentes perspetivas. Assim sendo, propor estratégias para Educação a Distância amplia as possibilidades de inovação e utilização de recursos tecnológicos e ambientes de aprendizagem. Através delas, é possível expandir possibilidades tanto para os professores, quanto para os alunos, além de gerar espaços de planeamento pedagógico, construção de conhecimento e compartilhamento de informações.

As estratégias pedagógicas, elaboradas neste estudo, abordaram especificamente aspetos sociais e afetivos, aprendizagem móvel e construção de MED pelo público idoso, contemplando diferentes contextos da EaD. A partir dos dados coletados, por meio do estudo de casos múltiplos, a presente pesquisa demonstrou, entre outras questões, que a construção de estratégias pedagógicas proporciona uma vasta gama de possibilidades na Educação a Distância, propiciando atividades inovadoras, com potencial para incluir os diferentes perfis de participantes e temáticas. Observou-se ainda a importância da realização da avaliação constante das estratégias desenvolvidas e aplicadas, já que o contexto pode modificar rapidamente, dependendo do feedback do aluno no decorrer do processo. Neste sentido, pretende-se, através deste artigo, fomentar uma discussão a respeito do conceito e da utilização de estratégias pedagógicas na EaD, assim como contribuir com a disseminação dessa prática na educação.

\section{REFERÊNCIAS}

Amaral, C. B. (2017). Estratégias pedagógicas para o ensino fundamental: Um enfoque na dimensão socioafetiva [Unpublished doctoral thesis]. Universidade Federal do Rio Grande do Sul, Porto Alegre, Brasil.

Bardin, L. (2010). Análise de Conteúdo. Edições 70.

Behar, P., Machado, L. R., Torrezzan, C. A. W., \& Longhi, M. T. (2019). Recomendação pedagógica em educação a distância: Conceitos e elementos. In P. A. Behar (Org.), Recomendação pedagógica em educação a distância (pp. 1-18). Penso.

Cavalcante Filho, A., Sales, V., \& Alves, F. (2020) Tutoria e identidade docente na educação a distância. Revista Pemo, 2(1), 1-15.

https://doi.org/10.47149/pemo.v2i1.3632.

Longaray, A. (2014) Estratégias para a Educação a Distância: um olhar a partir dos estados de ânimo do aluno [Unpublished master's thesis]. Universidade Federal do Rio Grande do Sul, Porto Alegre, Brasil.

Marques, A. M., Tanaka, L. H., \& Fóz, A. O. B. (2019). Avaliação de programas de intervenção para a aprendizagem socioemocional do professor: Uma revisão integrativa. Revista Portuguesa de Educação, 32(1), 35-51.

https://doi.org/10.21814/rpe.15133.

Masetto, M. T. (2003). Competência pedagógica do professor universitário.

Summus.

Matoski, A., Veiga, B. P., Silva, M. T. Q. S., Ribeiro, D. G. F., \& Alberti, M. E. (2020). Uso de dispositivos móveis como ferramenta de aprendizado: Riscos e oportunidades/ Use of mobile devices as a learning tool: Risks and opportunities. 
Brazilian Journal of Development, 6(1), 4673-4687. https://doi.org/10.34117/bjdv6n1335.

Meirelles, F. S. (2021). Pesquisa Anual do Uso de TI. FGV - Fundação Getúlio Vargas. https://eaesp.fgv.br/ensinoeconhecimento/centros/cia/pesquisa.

Ribeiro, A. C. R. (2019). MP-SocioAVA: modelo pedagógico com foco nas interações sociais em um ambiente virtual de aprendizagem [Unpublished doctoral thesis]. Universidade Federal do Rio Grande do Sul, Porto Alegre, Brasil.

Sales, G. L., Cunha, J. L. L., Gonçalves, A. J., Silva, J. B., \& Santos, R. L. (2017). Gamificação e Ensinagem Híbrida na Sala de Aula de Física: Metodologias Ativas Aplicadas aos Espaços de Aprendizagem e na Prática Docente. Conexões: Ciência e Tecnologia, 11(2), 45-52. https://doi.org/ 10.21439/conexoes.v11i2.1181.

Souza, P. (2012). Aprendizagem Colaborativa em Ambientes Virtuais de Aprendizagem. In C. Maciel (Org.), Ambiente Virtuais de Aprendizagem (pp. 121-159). MEC.

Souza, A.R., Sartori, A., \& Roesler, J. (2008). Mediação pedagógica na educação a distância: Entre enunciados teóricos e práticas construídas. Revista Diálogo Educacional, 8(24), 327-339. http://dx.doi.org/10.7213/rde.v8i24.3850.

Viana, J., \& Peralta, H. (2020). Aprender na era digital: Do currículo para todos ao currículo de cada um. Revista Portuguesa de Educação, 33(1), 137-157. https://doi.org/10.21814/rpe.18500.

\author{
Informação dos autores: \\ i Universidade Federal do Rio Grande do Sul, Brasil. \\ https: //orcid.org/0000-0003-4102-2225 \\ ii Universidade Federal do Rio Grande do Sul, Brasil. \\ https://orcid.org/0000-0001-8680-1105 \\ iii Universidade Federal do Rio Grande do Sul, Brasil. \\ https: //orcid.org/0000-0002-9238-1327 \\ iv Universidade Federal da Grande Dourados, Brasil. \\ https://orcid.org/0000-0002-4921-8399 \\ v Universidade Federal do Rio Grande do Sul, Brasil. \\ https://orcid.org/0000-0002-8277-7045 \\ vi Universidade Federal do Rio Grande do Sul, Brasil. \\ https: //orcid.org/0000-0002-1795-7541 \\ vii Universidade Federal do Rio Grande do Sul, Brasil. \\ https: //orcid.org/0000-0001-6791-311X \\ viii Universidade Federal do Rio Grande do Sul, Brasil. \\ https://orcid.org/0000-0001-6939-5678 \\ ix Universidade Federal do Rio Grande do Sul, Brasil. \\ https://orcid.org/0000-0002-8962-5466
}

Toda a correspondência relativa a este artigo deve ser enviada para:

Patricia Alejandra Behar

Av. Paulo Gama, 110 - Prédio 12105 - 3० andar sala 401, 90040060 - Porto Alegre (RS) - Brasil.

pbeharaterra.com.br

Recebido em 9 de setembro de 2019

Aceite para publicação em 7 de outubro de 2020 


\title{
Pedagogical strategies in distance education: A look from different contexts
}

\begin{abstract}
The goal of the present article is to present the mapping, creation and application of pedagogical strategies (PS) focused on Distance Education (DE) in different contexts. For that, extension courses were used in different areas, which dealt with socio-affective aspects of distance education, m-learning and digital inclusion of the elderly. The need of this investigation arises from the fact that the concept of pedagogical strategies is usually broad and often inaccurate. Although some studies do use the term, few authors discuss its meaning and application. Based on these, this article presents a definition of pedagogical strategies, as well as implementation situations in distance education. In this approach, PS can be considered as a set of actions put into practice by the teacher to achieve an education-aimed goal. The methodology used to carry out this research was a multiple case study of the qualitative type. Data collection was based on the development, application and evaluation of pedagogical strategies used in extension courses. From the analysis of the data, it was possible to map different strategies and verify the pertinence in their adequacy, according to the context and profile of each audience. Based on the studies we carried out, we can conclude that the pedagogical strategies need to be constantly evaluated, mainly due to the transformations that can occur with the inclusion of new digital technologies in the virtual context, in addition to the heterogeneous profile of the students.
\end{abstract}

Keywords: Pedagogical strategies;

Distance Education 


\section{Estrategias pedagógicas en la educación a distancia: Una mirada de diferentes contextos}

\section{RESUMEN}

El artículo tiene como objetivo presentar el mapeo, la construcción y la aplicación de estrategias pedagógicas (EP) centradas en la educación a distancia (ED) en diferentes contextos. Para ello, se utilizaron cursos de extensión en diferentes áreas, que trataron aspectos socio-afectivos de la educación a distancia, el aprendizaje virtual y la inclusión digital de las personas mayores. Se demostró que la investigación era necesaria en vista del hecho de que la comprensión del concepto de estrategias pedagógicas suele ser amplia $\mathrm{y}$, a menudo, imprecisa. Este artículo presenta una definición de estrategias pedagógicas, así como situaciones de implementación en educación a distancia. La educación física se puede considerar como un conjunto de acciones puestas en práctica por el profesor para lograr un objetivo dirigido a la educación. La metodología utilizada para llevar a cabo esta investigación fue el tipo cualitativo de estudio de caso múltiple. La recolección de datos se basó en el desarrollo, aplicación y evaluación de estrategias pedagógicas en cursos de extensión. A partir del análisis de los datos, fue posible mapear diferentes estrategias y verificar la pertinencia de su adecuación, de acuerdo con el contexto y el perfil de cada audiencia. La educación física debe ser evaluada constantemente, principalmente debido a las transformaciones que pueden ocurrir con la inclusión de nuevas tecnologías digitales en el contexto virtual, además del perfil heterogéneo de los estudiantes involucrados en el proceso educativo.

Palabras-clave: Estrategias pedagógicas; Educación a distancia 